\title{
Atorvastatin protects the proliferative ability of human umbilical vein endothelial cells inhibited by angiotensin II by changing mitochondrial energy metabolism
}

\author{
YE CHANG, YUAN LI, NING YE, XIAOFAN GUO, ZHAO LI, GUOZHE SUN and YINGXIAN SUN \\ Department of Cardiology, The First Hospital of China Medical University, Shenyang, Liaoning 110001, P.R. China
}

Received April 19, 2016; Accepted October 5, 2017

DOI: $10.3892 /$ ijmm.2017.3200

\begin{abstract}
This study aimed to explore whether angiotensin II (Ang II) inhibits the proliferation of human umbilical vein endothelial cells (HUVECs) by changing mitochondrial energy metabolism, and whether atorvastatin has a protective role via restoration of endothelial function. HUVECs were treated with $1 \mu \mathrm{M}$ Ang II alone or with $10 \mu \mathrm{M}$ atorvastatin for $24 \mathrm{~h}$. Proliferation was detected by MTT assay, cell counting, 5-ethynyl-2'-deoxyuridine assay and real-time cell analyzer. Mitochondrial energy metabolism including oxygen consumption rate and extracellular acidification rate were measured using a Seahorse metabolic flux analyzer. Mitochondrial membrane potential was detected under fluorescence microscope following staining with tetramethylrhodamine. Respiratory chain complexes I-V were detected using western blotting. The current study showed that Ang II inhibits the proliferation of HUVECs. Results from the Seahorse metabolic flux analyzer indicated that Ang II decreased basal oxygen consumption, maximal respiration capacity, spare respiration capacity, adenosine triphosphate-linked respiration and non-mitochondrial respiration. By contrast, Ang II increased the proton leak. Additionally, Ang II increased glycolysis, glycolytic capacity and non-glycolytic acidification. Furthermore, these effects were all suppressed by atorvastatin. The results indicated that atorvastatin prevents cellular energy metabolism switching from oxidative phosphorylation to glycolysis induced by Ang II and protected the proliferative ability of HUVECs.
\end{abstract}

\section{Introduction}

The renin-angiotensin-aldosterone system (RAAS) has an important role in the pathogenesis of cardiovascular diseases, including hypertension (1) and atherosclerosis (2). Angiotensin II (Ang II) is the main active peptide hormone of the RAAS and has a

Correspondence to: Dr Yingxian Sun, Department of Cardiology, The First Hospital of China Medical University, 155 North Nanjing Street, Heping, Shenyang, Liaoning 110001, P.R. China

E-mail:yxsun@mail.cmu.edu.cn

Key words: angiotensin II, proliferation, atorvastatin, mitochondria, human umbilical vein endothelial cell major role in endothelial dysfunction, vascular remodeling and vascular inflammation, which are closely associated with hypertension and atherosclerosis $(3,4)$. Previous study indicated that Ang II mediates an anti-growth effect via stimulation of the Ang II receptor type 2 in endothelial cells (5). Accumulating evidence demonstrated that Ang II could induce apoptosis of human umbilical vein endothelial cells (HUVECs) via a different pathway (6). However, there are limited studies on the effect of Ang II on the proliferation of HUVECs.

Mitochondria are the 'power plants' in cells that generate a large amount of adenosine triphosphate (ATP) to maintain normal cell function. Investigating the role of mitochondria in various cardiovascular diseases is currently an area of great interest (7). Mitochondrial damage and dysfunction has an important role in the atherosclerotic process, which seems to be reactive oxygen species (ROS)/reactive nitrogen species-dependent and may cause loss of bioenergetic control $(8,9)$. Mitochondria have a critical function in regulating the redox state, energy metabolism, proliferation, apoptosis and intracellular signaling $(10,11)$. In most studies, polarographic techniques have been used to examine mitochondria isolated from cells, however this methodology can not measure bioenergetic function in intact cells. Furthermore, this methodology has certain disadvantages, such as it can result in anoikis and increased oxidative stress (12). In this study, the effects of Ang II on cellular bioenergetic function were examined using a non-invasive technology, Seahorse Bioscience XF24 extracellular flux analyzer. This technology made it possible to determine the impact of Ang II on mitochondrial respiration and glycolysis in intact cells, and to characterize the changes in bioenergetics.

Statins, including atorvastatin, simvastatin, rosuvastatin and others, have exerted a variety of protective effects including upregulation of endothelial nitric oxide (NO) expression and antioxidant effects, which is independent of lowering cholesterol concentrations (13). Studies from both basic and clinical trial have demonstrated that atorvastatin has exhibited an antihypertensive effect by improving endothelial function, resisting oxidation and increasing vascular NO stores (14-16). However, further studies are required to elucidate the underlying mechanism how atorvastatin is involved in regulating stable endothelial function.

In the present study, it was aimed to explore whether Ang II inhibits proliferation of HUVECs by altering mitochondrial 
energy metabolism and whether atorvastatin has a protective role via restoration of endothelial function.

\section{Materials and methods}

Cell culture and treatments. HUVECs (obtained from the Cell Bank of the Chinese Academy of Sciences, Shanghai, China) were cultured in Dulbecco's modified Eagle's medium (DMEM; Hyclone; GE Healthcare Life Sciences, Logan, UT, USA) containing $10 \%$ fetal bovine serum (FBS; Hyclone; GE Healthcare Life Sciences) and penicillin-streptomycin (100 U/ml-100 $\mu \mathrm{g} / \mathrm{ml}$; Sigma-Aldrich; Merck KGaA, Darmstadt, Germany), in humidified atmosphere with $5 \% \mathrm{CO}_{2}$ at $37^{\circ} \mathrm{C}$. Medium was replaced every 2 days or as necessary. For each experiment, the cells were treated with $1 \mu \mathrm{M}$ Ang II alone or in combination with $10 \mu \mathrm{M}$ atorvastatin (both from Sigma-Aldrich; Merck KGaA) for 24 h. Trypsin (0.25\%) (Sigma-Aldrich; Merck KGaA) was used for digestion.

Cell viability analysis. Cell viability was determined by measuring the metabolism of 3-(4,5-dimethylthiazol-2-yl)2,5-diphenyltetrazolium bromide (MTT), as described in our previous study (17). Briefly, HUVECs were plated in a 96-well plate at density of 2,000 cells/well. The medium was changed after 1 day. The cells were incubated with fresh medium containing $1 \mu \mathrm{M}$ Ang II alone or with $10 \mu \mathrm{M}$ atorvastatin for $24 \mathrm{~h}$. Subsequently, $10 \mu \mathrm{l}$ sterile MTT solution (final concentration, $0.5 \mathrm{mg} / \mathrm{ml}$ ) was added to each well. After incubation at $37^{\circ} \mathrm{C}$ for $4 \mathrm{~h}$ in the dark, the culture media containing MTT was removed and replaced with $100 \mu 1 \mathrm{DMSO}$. The plate was gently rotated on a linear and orbital shaker for 3-5 min to completely dissolve the precipitation. The absorbance was measured with microplate reader (Bio-Rad Laboratories, Inc., Hercules, CA, USA) at $570 \mathrm{~nm}$. The percentage of cell viability was calculated according to the following formula: Cell viability $(\%)=$ optical density $(\mathrm{OD})$ of the treatment group/OD of the control group $\times 100$.

Cell proliferation assay. Cells were seeded into 6-well plates at a density of 10,000 cells/well in $2 \mathrm{ml}$ medium supplemented with $10 \%$ FBS. The medium was changed every 2 days or in necessary. Cell number at the indicated time-points (1, 2, 3 and 4 days) was determined by counting using a hemocytometer.

Growth curve assays using real-time cell analyzer (RTCA). To analyze the cell proliferation continuously over time, growth curve assays were performed in RTCA in quadruplicate with the xCELLigence system (ACEA Bioscience, Inc. San Diego, CA, USA) according to the methods as previously described (18). Briefly, 5,000 cells/well were seeded in RTCA E-plates (ACEA Bioscience, Inc.), the electrical impedance in each well was measured continuously. The shift of the electrical impedance was expressed as the cell index, which was a parameter of cell viability.

5-ethynyl-2'-deoxyuridine (EdU) incorporation analysis. As described by our previous studies $(17,19)$ and the manufacturer's instructions, the DNA synthesis rate in HUVECs was determined by an EdU incorporation analysis using Click-iT ${ }^{\mathrm{TM}}$ EdU Alexa Fluor 555 Imaging kit (Invitrogen; Thermo Fisher
Scientific, Inc., Waltham, MA, USA). Briefly, the cells were incubated with EdU-labeling solution for $8 \mathrm{~h}$ at $37^{\circ} \mathrm{C}$, and then fixed with $4 \%$ cold formaldehyde for $30 \mathrm{~min}$ at room temperature. After permeabilization with 1\% Triton X-100 for $20 \mathrm{~min}$, the cells were reacted with Click-iT reaction cocktails (Invitrogen; Thermo Fisher Scientific, Inc.) for $30 \mathrm{~min}$ at room temperature. Subsequently, the DNA contents of the cells were stained with Hoechst 33342 (final concentration, $5 \mu \mathrm{g} / \mathrm{ml}$ ) for $30 \mathrm{~min}$. Finally, EdU-labeled cells were counted using ImageJ 1.47 software (National Institutes of Health, Bethesda, MD, USA) and normalized to the total number of Hoechst-stained cells. At least 500 cells each experiment were counted, EdU-positive cells were expressed as a percentage of the total cells.

Western blotting. Western blotting was performed as previously described $(17,20)$. The cells were lysed in lysis buffer $(20 \mathrm{mM}$ Tris- $\mathrm{HCl}, 150 \mathrm{mM} \mathrm{NaCl}, 2 \mathrm{mM}$ EDTA and 1\% Triton X-100) containing a protease inhibitor cocktail (Sigma-Aldrich; Merck KGaA). The total protein concentrations were measured by a BCA Protein Assay kit (Sigma-Aldrich; Merck $\mathrm{KGaA})$. After heat-denaturing, equal quantities of proteins $(20 \mu \mathrm{g})$ were separated by NuPAGE Novex 12\% Bis-Tris Gel and electrophoresed in the XCell SureLock ${ }^{\mathrm{TM}}$ Mini-Cell (both from Invitrogen; Thermo Fisher Scientific, Inc.), and then transferred to polyvinylidene difluoride membranes and blocked with 5\% non-fat milk in Tris-buffered saline (TBS) for $1.5 \mathrm{~h}$ at the room temperature. The membranes were incubated with primary antibodies against OXPHOS (ab110413; 1:1,000; Abcam, Cambridge, UK) or GAPDH (AB2302; 1:1,000; EMD Millipore, Billerica, MA, USA) overnight at $4^{\circ} \mathrm{C}$. After washing three times with $1 \mathrm{X}$ TBS, the membranes were incubated with secondary antibodies [anti-mouse (AP181R) and anti-rabbit antibodies (AP187R); 1:10,333; EMD Millipore] for $1.5 \mathrm{~h}$ at room temperature. After washing steps, immunoreactive binding was detected with ECL detection reagent (Amersham Biosciences, Piscataway, NJ, USA) with MicroChemi 4.2. The band intensity was quantified using ImageJ 1.47 software.

Measurement of mitochondrial membrane potential. We used tetramethylrhodamine (TMRE; Molecular Probes; Thermo Fisher Scientific, Inc.) to detect changes in mitochondrial membrane potential, as described previously $(21,22)$. Briefly, unfixed live cells were incubated with $100 \mathrm{nM}$ TMRE in the dark for $30 \mathrm{~min}$ at $37^{\circ} \mathrm{C}$ and $5 \% \mathrm{CO}_{2}$. After washing, cells were images using a fluorescence microscope.

Bioenergetic measurements using Seahorse mitochondrial flux analyses. To measure the rate of oxidative phosphorylation (OXPHOS) and glycolysis in HUVECs, a Seahorse metabolic flux analyzer was used, according to methods previously described $(12,23)$. For measuring oxygen consumption rate (OCR), XF Cell Mito Stress Test kit (Agilent Technologies, Inc., Santa Clara, CA, USA) including different pharmacological inhibitors were used to probe the function of individual components in the respiratory chain. The cells were incubated in XF24 culture microplates with culture medium for $24 \mathrm{~h}$ as well as the sensor cartridge hydrated in XF Calibrant at $37^{\circ} \mathrm{C}$ in a non- $\mathrm{CO}_{2}$ incubator overnight. Prior to all bioenergetic assays, the culture medium was replaced $1 \mathrm{~h}$ before with unbuffered DMEM (pH 7.4) supplemented with $4 \mathrm{mM}$ L-glutamine. To estimate the 
basal OCR coupled to ATP synthesis, $1 \mu \mathrm{M}$ oligomycin was injected to inhibit the ATP synthase (complex V). Typically, the decreased OCR in response to oligomycin indicated the cells were using mitochondria to generate ATP. The remaining OCR could be ascribed to either proton leakage or the demand on the mitochondrial proton gradient for the movement of ions or metabolites. To determine the maximal OCR that the cells could sustain, $0.5 \mu \mathrm{M}$ carbonyl cyanide-4-(trifluoromethoxy) phenylhydrazone (FCCP) was injected, which made the mitochondrial inner membrane permeable to protons. Finally, $0.5 \mu \mathrm{M}$ antimycin A (Anti-A) and rotenone was injected to inhibit electron flux. The remaining OCR could be ascribed to oxygen consumption due to the formation of mitochondrial ROS and non-mitochondrial sources.

For measuring extracellular acidification rate (ECAR), XF Glycolysis Stress Test kit (Agilent Technologies, Inc.) including different pharmacological inhibitors were used to probe the function of glycolysis. Initially, cells were incubated in sugar or pyruvate-free glycolytic assay medium for $1 \mathrm{~h}$ before the assay and then the first injection of a saturating concentration of glucose was conducted. The cells may catabolize glucose into pyruvate via glycolysis pathway, producing ATP, NADH, water and protons. The extrusion of protons into surrounding medium leaded to a sudden increase in ECAR, which was defined as basal glycolytic capacity. The second injection was oligomycin that could shift energy production to glycolysis by means of restraining mitochondrial ATP production. Consequently, the sharp increase of ECAR indicated the level of glycolytic capacity. The final injection was 2-deoxy-D-glucose, a glucose analog, which inhibited glycolysis through competitive binding to glucose hexokinase, the first enzyme in the glycolytic pathway. The decrease in ECAR confirmed that the ECAR produced in the experiment was caused by glycolysis. The gap between glycolytic capacity and glycolysis was defined as glycolytic reserve. ECAR, prior to glucose injection was referred as non-glycolytic acidification and that may due to other processes in cells.

First, the optimum number of cells needed for these experiments was determined. HUVECs were seeded to a density of $10,000,20,000,40,000$ or 80,000 cells/well. Oxygen consumption in these cells was proportional to cell number within this range, and a seeding density of 10,000 cells/well was selected for the subsequent experiments. Subsequently, an assay was developed to measure indices of mitochondrial function in HUVECs incubated with Ang II and/or atorvastatin. HUVECs were seeded at a density of 10,000 cells/well in the 24-well Seahorse assay plates. Cells were then serum-starved in 0.1\% FBS DMEM for $24 \mathrm{~h}$ and subsequently $1 \mu \mathrm{M}$ Ang II alone or with $10 \mu \mathrm{M}$ atorvastatin were added for $24 \mathrm{~h}$. Medium was replaced with DMEM and then incubated for $1 \mathrm{~h}$ at $37^{\circ} \mathrm{C}$ without $\mathrm{CO}_{2}$. XF Cell Mito Stress Test kit was used to test the mitochondrial respiration and XF Glycolysis Stress Test kit (Agilent Technologies, Inc.) was used to test glycolysis function. To allow comparison between experiments, data are expressed as the rate of OCR in $\mathrm{pmol} / \mathrm{min}$ or the rate of ECAR in $\mathrm{mpH} / \mathrm{min}$.

Statistical analysis. The statistical significance of the difference was analyzed by analysis of variance and post hoc Dunnett's test. All experiments were repeated for at least three times, and data were expressed as the mean \pm standard deviation. All analyses were performed using SPSS 17.0 statistical software (SPSS, Inc., Chicago, IL, USA) and $\mathrm{P}<0.05$ was considered to indicate a statistically significant difference.

\section{Results}

Atorvastatin attenuates the Ang II-induced inhibition of the proliferation of HUVECs. To investigate how atorvastatin affected Ang II-inhibited proliferation in HUVECs, cells were cultured with $1 \mu \mathrm{M}$ Ang II alone or with $10 \mu \mathrm{M}$ atorvastatin for $24 \mathrm{~h}$. An MTT assay to measure the cellular activity, and the proliferation level was determined by cell counting, EdU staining and RTCA. As presented in Fig. 1, $1 \mu \mathrm{M}$ Ang II exhibited an inhibitory effect on cellular activity (Fig. 1A) and the proliferation of HUVECs (Fig. 1B-D). Co-treatment with $10 \mu \mathrm{M}$ atorvastatin recovered cellular activity, and similar results were observed with the proliferation level. The result was further confirmed by the results of RTCA (Fig. 1E). Therefore, these results suggested that atorvastatin attenuated Ang II-induced inhibition of proliferation in HUVECs.

Measurement of mitochondrial function in endothelial cells. To assess cellular bioenergetics in intact endothelial cells, the Seahorse metabolic flux analyzer was used to determine rates of oxygen consumption and glycolysis $(12,23)$. The flow charts of the experimental design are presented in Fig. 2A and B, and the experimental principles and methods are detailed described in the Materials and methods. In the first series of experiments, the optimal number of HUVECs required to obtain measurable OCR and ECAR was established, as shown in Fig. 2C and D. Both OCR and ECAR responses were proportional to cell number. For subsequent experiments, a seeding density of 10,000 cells/well was selected to detect the changes in OCR and ECAR due to exposure to Ang II and/or atorvastatin.

Effect of Ang II and/or atorvastatin on mitochondrial aerobic metabolism. The inhibitory effects of statins on Ang II-induced endothelial dysfunction has described previously (24). Although statins played a protective effect for endothelial dysfunction induced by Ang II, the underlying mechanism was still not clear. We speculate that it may be associated with cellular energy metabolism.

To further investigate the changes of mitochondrial aerobic metabolism that occurred in HUVECs in response to Ang II and/or atorvastatin, a Cell Mito Stress assay kit was used to detect the OCR. HUVECs were treated with $1 \mu \mathrm{M}$ Ang II and/or $10 \mu \mathrm{M}$ atorvastatin for $24 \mathrm{~h}$ before exposure to $1 \mu \mathrm{M}$ oligomycin, $0.5 \mu \mathrm{M}$ FCCP and $0.5 \mu \mathrm{M}$ rotenone and Anti-A. As demonstrated in Fig. 3A, Ang II reduced the OCR in HUVECs, suggesting that Ang II inhibits mitochondrial aerobic respiration. These data are used to calculate an apparent respiratory control ratio (RCR) for various metabolic conditions for mitochondria in cells (12).

$$
\begin{aligned}
& \mathrm{RCR}_{\text {basal }}=(\text { basal }- \text { Anti-A }) /(\text { Oligo }- \text { Anti-A }) \\
& \mathrm{RCR}_{\text {max }}=(\mathrm{FCCP}-\text { Anti-A }) /(\text { Oligo }- \text { Anti-A })
\end{aligned}
$$

RCR under the basal condition was $10.0 \pm 1.8$ and under maximal condition was $22.1 \pm 4.3$ at a seeding density of 10,000 cells/well, which indicated that the mitochondria were 
A

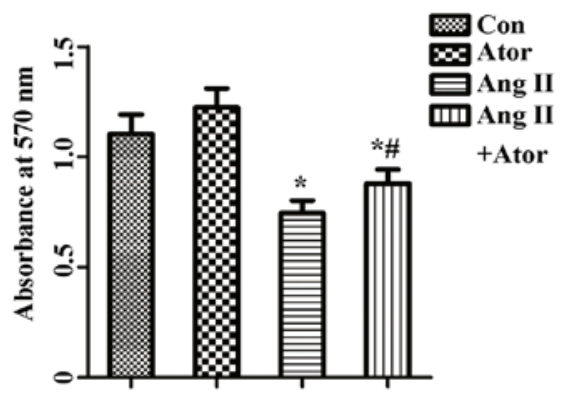

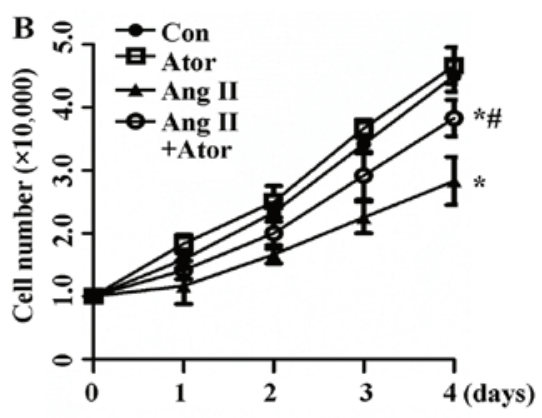

C
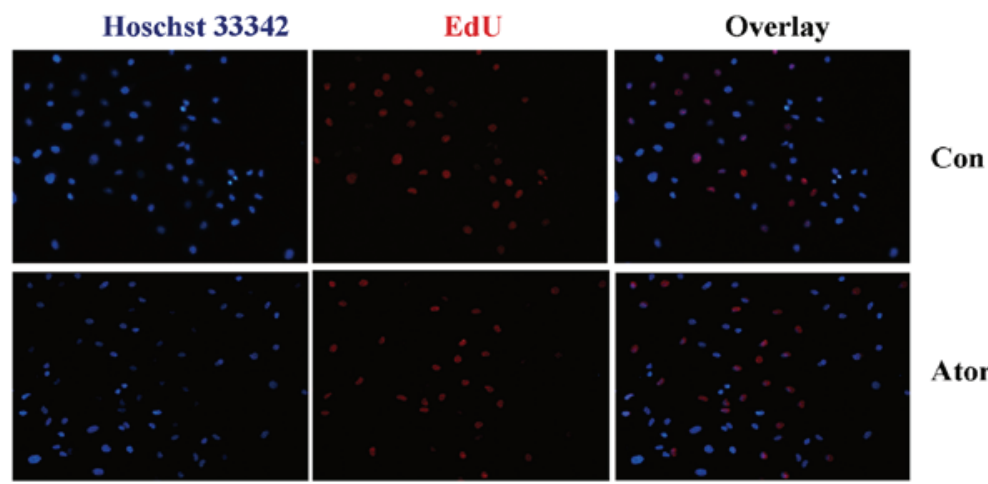

Ator
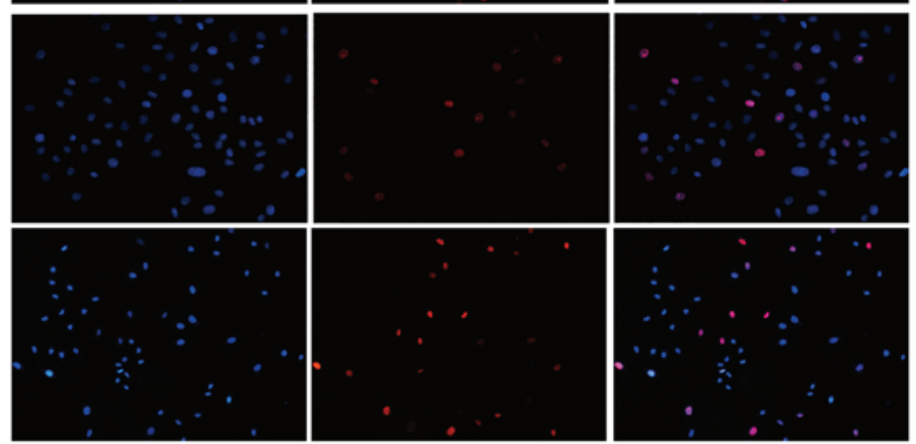

Ang II

Ang II+Ator

D
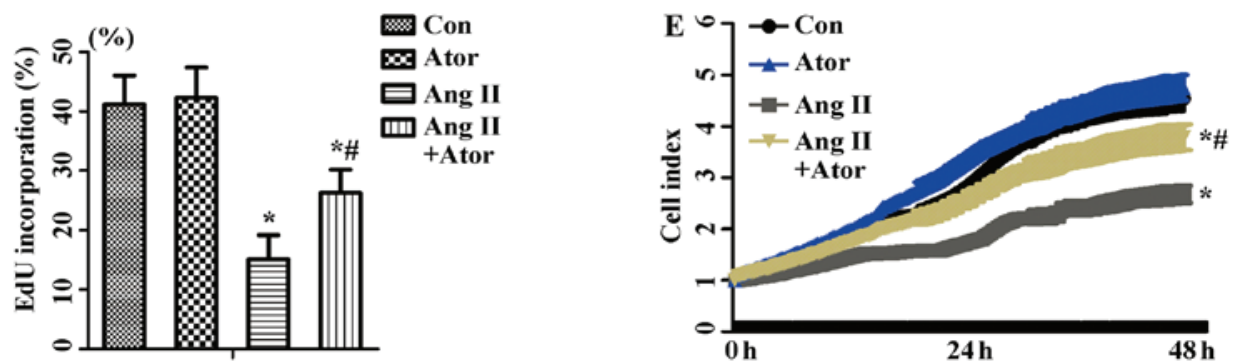

Figure 1. Atorvastatin attenuated Ang II-inhibited proliferation in human umbilical vein endothelial cells. Cells were cultured in the presence of $1 \mu \mathrm{M}$ Ang II and/or $10 \mu \mathrm{M}$ atorvastatin. (A) Cell viability was detected by MTT assay. (B) Cells were plated on 6-well plate and cell numbers were counted daily for 4 days (C) Cell proliferation was detected by EdU assay and the red staining represents the new cells and (D) quantified by EdU incorporation (\%). (E) Cell index was measured by real-time cell analyzer. Results are presented as the mean \pm standard deviation of at least three experiments. " $\mathrm{P}<0.05$ vs. control; ${ }^{\prime} \mathrm{P}<0.05$ vs. Ang II. Con, control; Ator, atorvastatin; Ang II, angiotensin II; EdU, 5-ethynyl-2'-deoxyuridine.

tightly coupled under normal physiological conditions in HUVECs. Additionally, Ang II decreased the RCR (Fig. 3B). Furthermore, Ang II decreased basal oxygen consumption, maximal respiration capacity, spare respiration capacity, ATP-linked respiration and non-mitochondrial respiration compared with the control group (Fig. 3C-F and H). By contrast, Ang II increased the proton leak (Fig. 3G). Furthermore, atorvastatin significantly reversed the dysfunction of aerobic metabolism in mitochondria induced by Ang II (Fig. 3).

Effect of Ang II and/or atorvastatin on glycolytic function. In addition to OCR, the Seahorse metabolic flux analyzer can be used to measure the protons produced by the cells, and the ECAR indicated lactate production and was therefore an index 

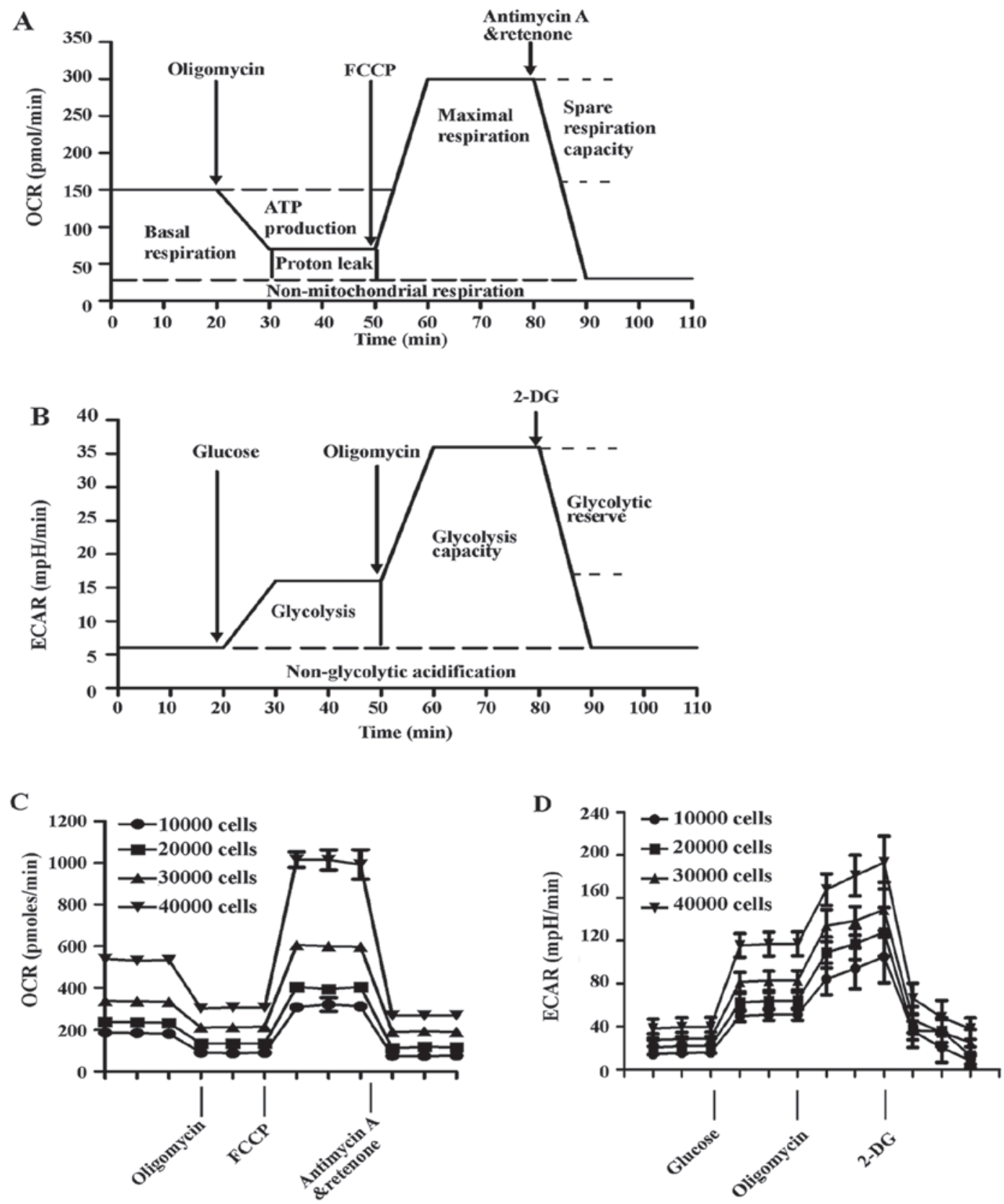

Figure 2. Measurement of mitochondrial function in HUVECs. The experiments were performed using the XF24 extracellular flux analyzer, and the flow chart showed the measurement of (A) OCR and (B) ECAR. The optimal number $(10,000,20,000,40,000$ and 80,000 cells/well) of HUVECs were seeded in the Seahorse Bioscience microplates. (C) OCR and (D) ECAR were measured and plotted as a function of cell seeding number. Results are presented as the mean \pm standard deviation $(\mathrm{n}=4)$. HUVECs, human umbilical vein endothelial cells; OCR, oxygen consumption rate; FCCP, carbonyl cyanide-4-(trifluoromethoxy)phenylhydrazone; ECAR, extracellular acidification rate; 2-DG, 2-deoxy-D-glucose.

of glycolysis (25). As shown in Fig. 4A, ECAR increased with the inhibition of Ang II on mitochondrial respiration, which was attributable to the increased requirement for glycolysis to generate ATP. The parameters of glycolytic function included glycolysis, glycolytic capacity, glycolytic reserve and non-glycolytic acidification. Following quantification, all the parameters of glycolytic function besides glycolytic reserve were increased in the Ang II-treatment group compared with the control treatment group (Fig. 4B-E). Furthermore, atorvastatin significantly reversed the glycolysis dysfunction induced by Ang II.

Effect of Ang II and/or atorvastatin on mitochondrial membrane potential and mitochondrial respiration chain complexes. To determine whether Ang II and/or atorvastatin had effects on mitochondrial membrane potential, HUVECs were labeled with TMRE (red) and the nuclei were stained with Hoechst 33342 (blue). TMRE (red) staining indicated that mitochondrial membrane potential was reduced by Ang II; however co-treatment with atorvastatin maintained the mitochondrial normal membrane potential (Fig. 5A).

In order to investigate the effect of Ang II on mitochondrial respiration chain complexes, western blotting was performed to detect the protein level of OXPHOS components, including complexes I-V. The expression of complexes I-V was markedly decreased in the Ang II group compared with the control treatment (Fig. 5B and C). Furthermore, co-treatment with Ang II 
A

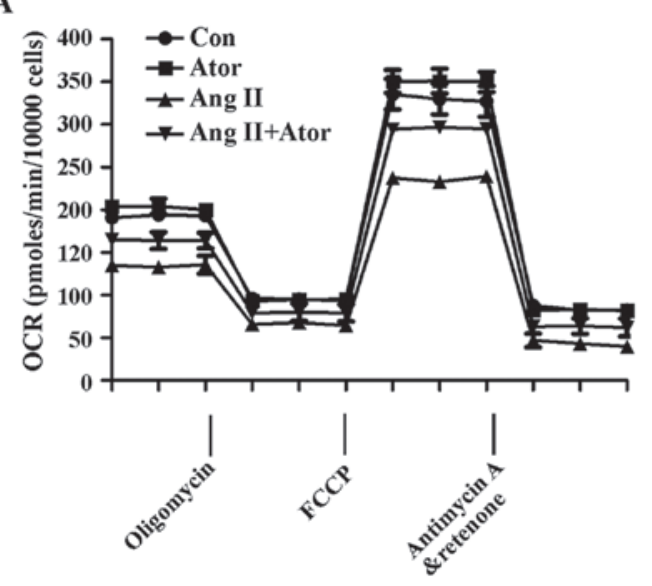

B

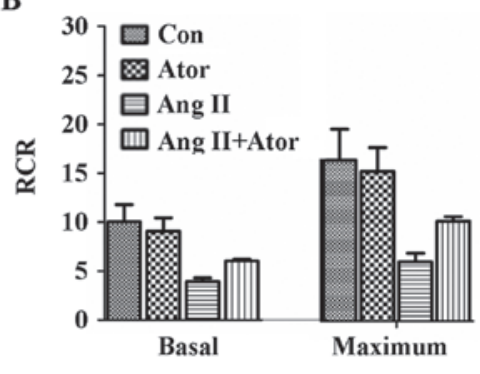

C

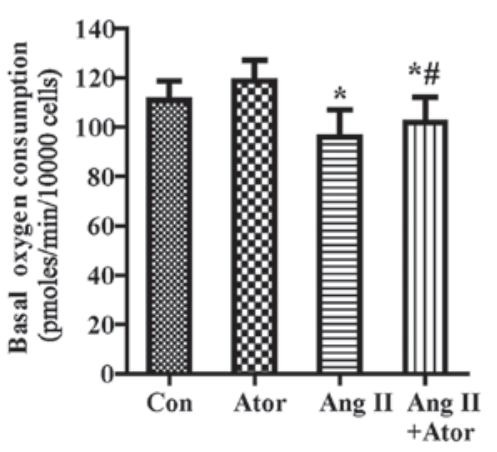

E

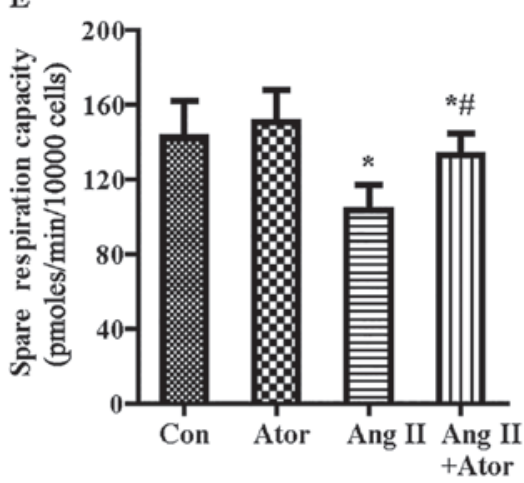

G

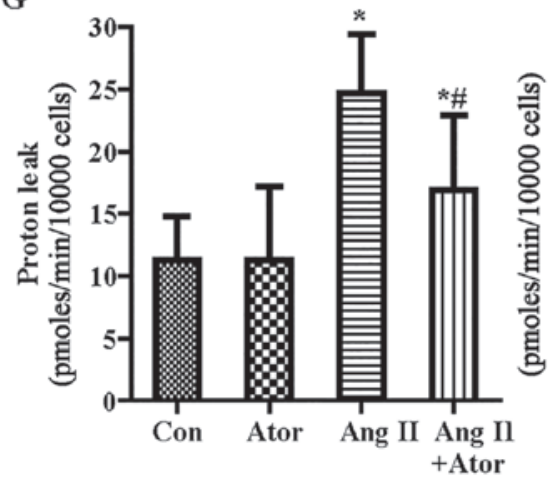

D

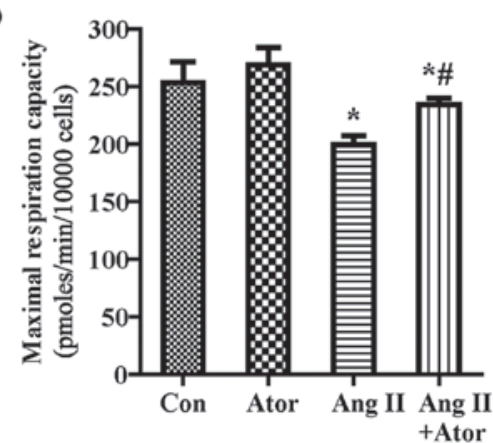

F

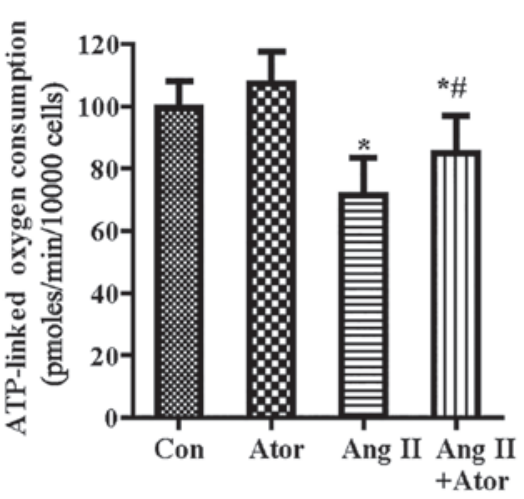

H

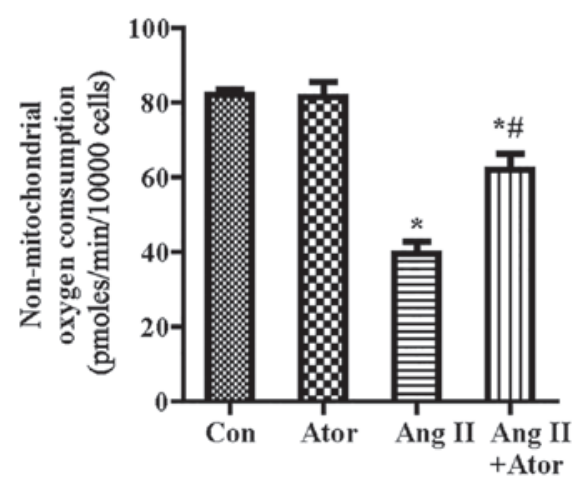

Figure 3. Effect of Ang II and/or atorvastatin on mitochondrial aerobic metabolism in HUVECs. HUVECs were seeded in the Seahorse Bioscience microplates (10,000 cells/well). (A) The OCR was conducted using Mito Stress Test kit. After adherence for $4 \mathrm{~h}, 1 \mu \mathrm{M}$ Ang II and/or $10 \mu \mathrm{M}$ atorvastatin was added into the microplates for co-incubation with cells for $24 \mathrm{~h}$ with subsequent injection of $1 \mu \mathrm{M}$ oligomycin, $0.5 \mu \mathrm{M}$ FCCP and $0.5 \mu \mathrm{M}$ rotenone and antimycin A. (B) The contribution of associated parameters including RCR, (C) basal oxygen consumption, (D) maximal oxygen consumption, (E) spare respiration capacity, (F) ATP-linked oxygen consumption, (G) proton leak and (H) non-mitochondrial oxygen consumption to the total cellular oxygen consumption was plotted, respectively. Each data point represented an OCR measurement. Results shown represent mean \pm standard deviation ( $\mathrm{n}=4)$. ${ }^{*} \mathrm{P}<0.05 \mathrm{vs}$. control; ${ }^{\#} \mathrm{P}<0.05$ vs. Ang II. HUVECs, human umbilical vein endothelial cells; Con, control; Ang II, angiotensin II; Ator, atorvastatin; OCR, oxygen consumption rate; RCR, respiratory control ratio. 

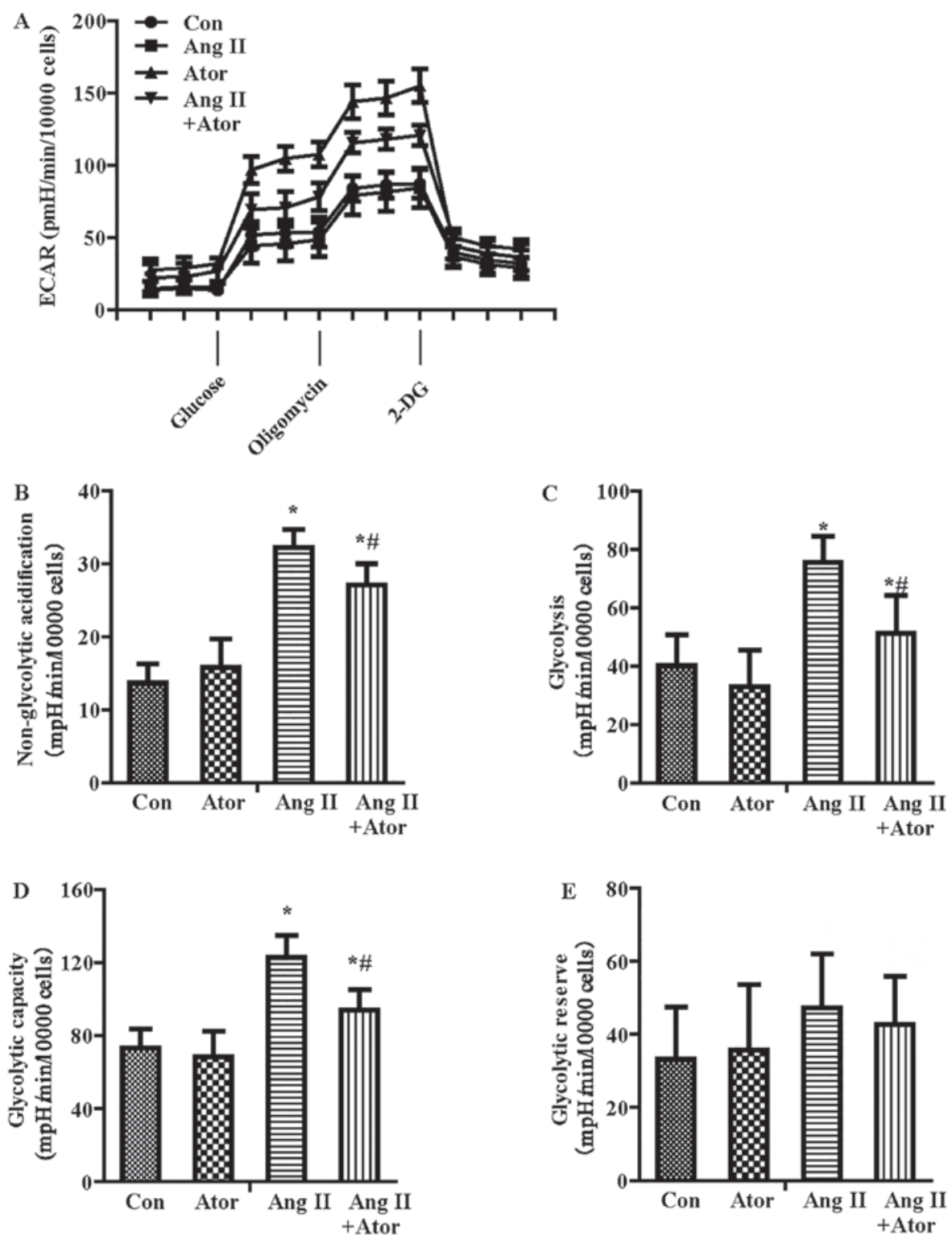

Figure 4.Effect of Ang II and/or atorvastatin on glycolytic function in HUVECs. HUVECs were seeded in the Seahorse Bioscience microplates (10,000 cells/well). (A) The ECAR was conducted using XF Glycolysis Stress Test kit. After adherence for $4 \mathrm{~h}, 1 \mu \mathrm{M}$ Ang II and/or $10 \mu \mathrm{M}$ atorvastatin was added into the microplates for co-incubation with cells for $24 \mathrm{~h}$ with subsequent injection of $15 \mathrm{mM}$ glucose, $2 \mu \mathrm{M}$ oligomycin and $50 \mathrm{mM}$ 2-DG. (B) The contribution of associated parameters including non-glycolytic acidification, (C) glycolysis, (D) glycolytic capacity and (E) glycolytic reserve to the total ECAR was plotted, respectively. Each data point represented an ECAR measurement. Results shown represent mean \pm standard deviation $(n=4)$. " $\mathrm{P}<0.05$ vs. control; ${ }^{~} \mathrm{P}<0.05$ vs. Ang II. HUVECs, human umbilical vein endothelial cells; ECAR, extracellular acidification rate; Con, control; Ang II, angiotensin II; Ator, atorvastatin; 2-DG, 2-deoxy-D-glucose.

and atorvastatin rescued the inhibition in OXPHOS protein expression induced by Ang II.

\section{Discussion}

In the present study, a newly emerging assay was applied to determine the effect of Ang II and atorvastatin on mitochondrial function in HUVECs. The principal findings of this study demonstrated that atorvastatin attenuated the inhibitory effect of Ang II on the proliferation of HUVECs via the mitochondria.

Under physiological conditions, endothelial cells maintain low proliferation capacity to reduced the damage of shear stress caused by blood flow. Ang II is the main active peptide hormone of the RAAS and has a major role in hypertension and atherosclerosis $(3,4)$. A previous study demonstrated that Ang II could induce apoptosis of HUVECs via different pathway (6). However, there are limited studies on the effect of Ang II on the proliferation of HUVECs. To the best of our knowledge, the current study is the first to demonstrate that Ang II inhibits the proliferation of HUVECs by damaging mitochondria to decrease aerobic metabolism, and making energy conversion to glycolysis. To regulate redox state, energy metabolism, apoptosis and intracellular signaling, cells need large amounts of ATP, which is predominantly generated by mitochondria $(10,11)$. Glucose metabolism and glycolysis are two important sources of ATP in cells. Notably, the calculated RCR values in HUVECs indicated that the mitochondria were tightly coupled under normal physiological conditions. However, Ang II treatment of HUVECs decreased RCR, which affected the coupling efficiency of OXPHOS. In addi- 
A
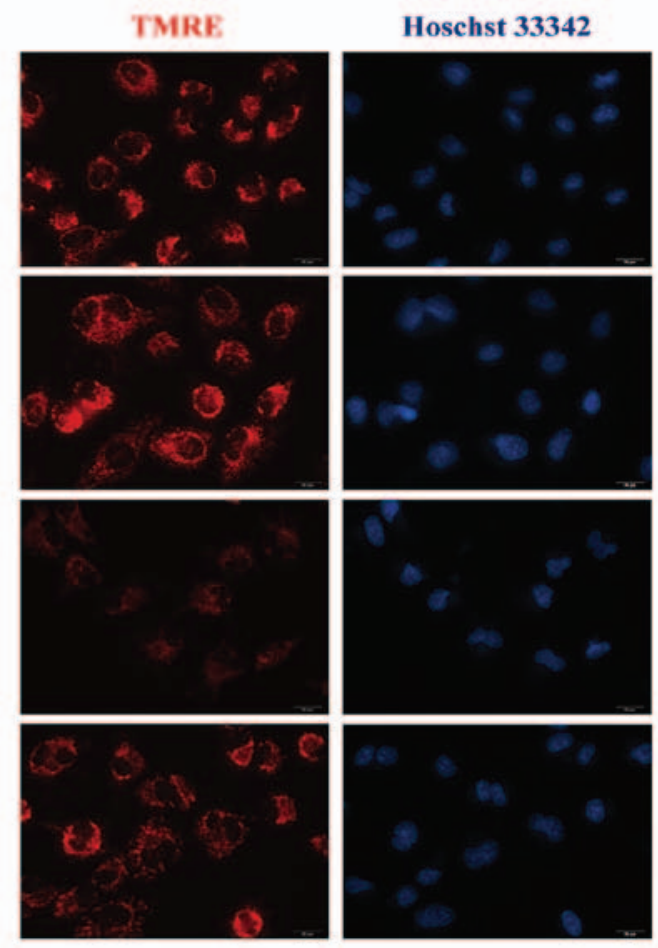

Hoschst 33342
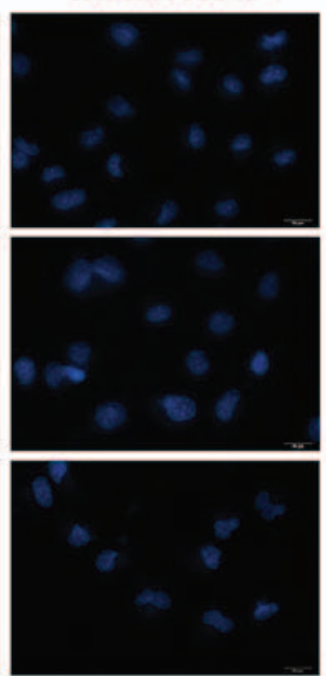

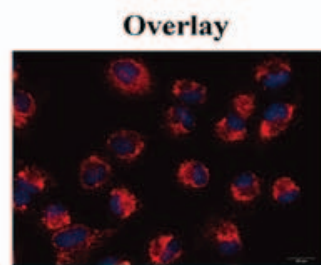

Con

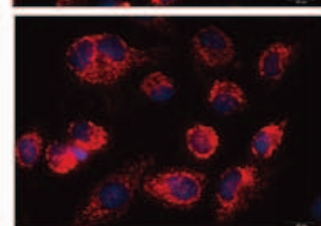

Ator

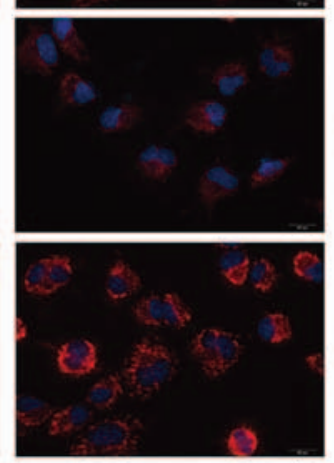

Ang II

Ang II+Ator

B
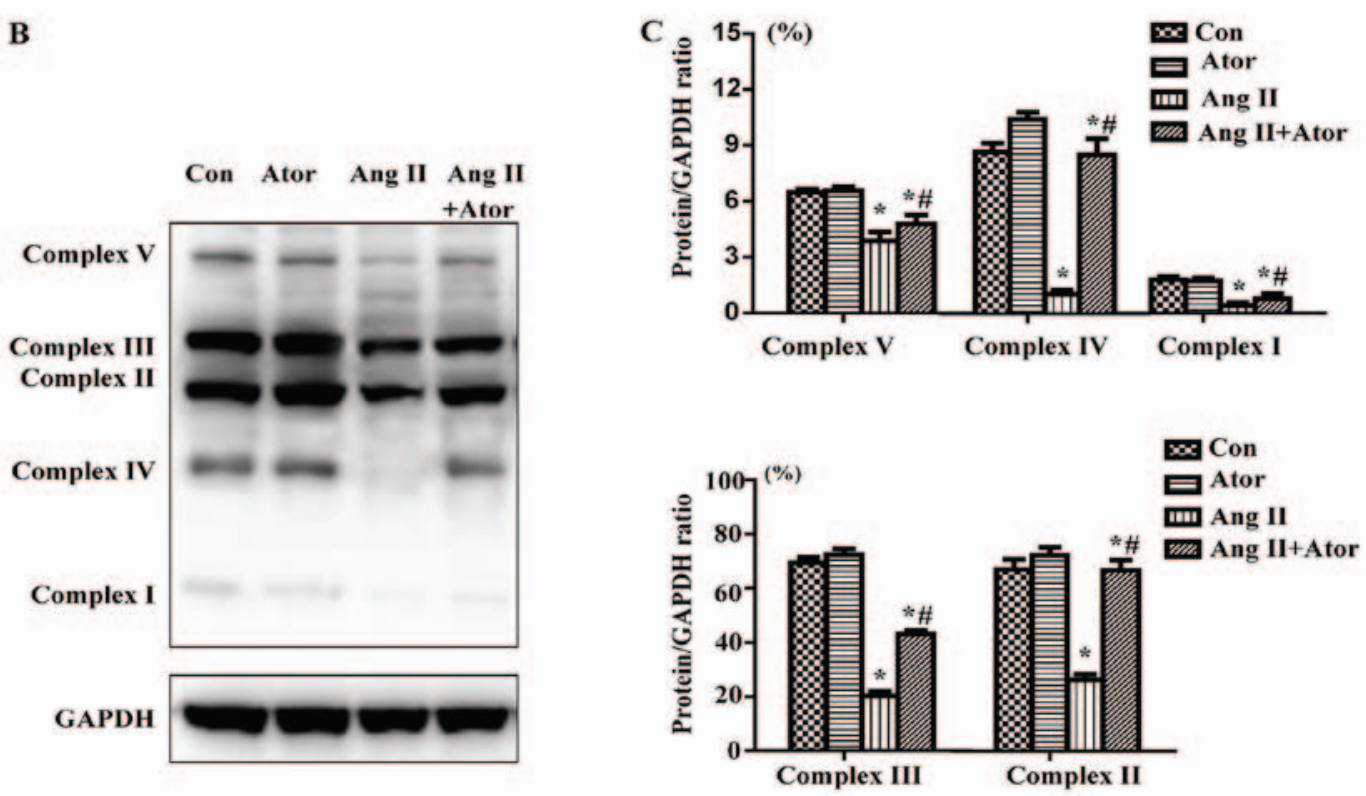

Figure 5. Effect of Ang II and/or atorvastatin on mitochondrial membrane potential and mitochondrial respiration chain complexes in HUVECs. (A) The mitochondria of the HUVECs were marked with TMRE (red), and the HUVECs nuclei were stained with Hoechst 33342 (blue). (B) Mitochondrial respiration chain complexes I-V were detected by western blotting and GAPDH served as a control, and (C) the band intensity was quantified using Image J 1.47 software, normalized by the control. Results are presented as the mean \pm standard deviation for at least three experiments. "P<0.05 vs. control; " $\mathrm{P}<0.05$ vs. Ang II. HUVECs, human umbilical vein endothelial cells; TMRE, tetramethylrhodamine; Con, control; Ator, atorvastatin; Ang II, angiotensin II.

tion, Ang II decreased the basal and ATP-linked oxygen consumption, which may decrease the generation of ATP necessary to the proliferation of HUVECs. Furthermore, loss of mitochondrial maximal and reverse respiration capacity lead to a decrease ability to respond to secondary energetic stressors, and failure to maintain organ function and cellular repair (12,26). Additionally, Ang II increased proton leak and lead to the collapse of mitochondrial membrane potential. The increased level of proton leak across the inner mitochondrial membrane is partially dependent on uncoupling proteins or leakage through damaged respiratory complexes (7). In addition, Ang II could reduced the expression of OXPHOS proteins (i.e., respiratory complexes including complexes I-V). Dysfunction in the mitochondrial respiration chain caused reduced basal respiration capacity, reduced ATP-linked oxygen consumption and s reduced pare respiration capacity, resulting in a decreased in ATP production (26). Previous studies demonstrated that through Ang II receptor, Ang II 
was implicated in the generation of ROS, the accumulation of which may cause mitochondrial dysfunction in HUVECs (27). On the other hand, inhibition of the electron transport chain in mitochondria can increase ROS generation (26). Therefore, mitochondrial dysfunction and ROS may promote each other in a feedback look and ultimately lead to cell death.

A previous study demonstrated that high glycolytic flux could yield more ATP in a short time than OXPHOS when energy supply is deficient in cells (28). Other studies also proved that the stress injury of stimuli significantly augmented glycolysis response to the decreased OCR in myocardial cells or bovine aortic endothelial cells $(7,12)$. Consistently, the results of the present study indicated that Ang II stimulated ECAR in HUVECs in response to energy demand and expenditure, which caused energy metabolism to switch from OXPHOS to glycolysis.

Studies from both basic research and clinical trials have demonstrated that atorvastatin has an antihypertensive effect by improving endothelial function, resisting oxidation and increasing vascular NO stores (14-16). Notably, there are also studies that indicate that stains had side-effects in skeletal muscle, ranging from mild weakness to severe rhabdomyolysis $(29,30)$. It is well-known that statins competitively inhibit 3-hydroxy-3-methylglutaryl-coenzyme (HMG-CoA) reductase, which catalyzes the rate limiting step in cholesterol biosynthesis (30). With the inhibition of HMG-CoA reductase, ubiquinol biosynthesis is also suppressed, which is an essential component of normal mitochondrial electron transport (31). Ubiquinol reduction may contribute to rhabdomyolysis. Furthermore, a previous study indicated that treatment with simvastatin reduces mitochondrial content, cell metabolism and cell viability in muscle cells (32). Furthermore, treatment with simvastatin significantly suppresses basal oxidative reliance in muscle cells (32). Notably, simvastatin had no effect on oxygen consumption in HepG2 cells (33). The difference in susceptibility to toxicity of simvastatin between liver HepG2 cells and skeletal muscle C2C12 myotubes was associated with the activity of the insulin-like growth factor-1/Akt signaling pathway (33). A previous study indicated that compared with hydrophilic rosuvastatin, lipophilic atorvastatin and simvastatin had a more pronounced effect on ubiquinol production in muscle tissue (34). However, the results of the current study indicated that atorvastatin had no toxicity on the mitochondrial aerobic metabolism, mitochondrial membrane potential and mitochondrial respiration chain complexes. In addition, atorvastatin attenuated Ang II-induced mitochondrial dysfunction. This difference in tolerance to toxicity of stains may reflect a difference in the activity of certain signaling pathways in different cell lines.

The current study focused on the protective effect of atorvastatin on Ang II-inhibited proliferation of HUVECs. Investigation into cellular bioenergetics demonstrated that atorvastatin prevented cellular energy metabolism switching from OXPHOS to glycolysis induced by Ang II. Atorvastatin may attenuate the Ang-II-induced damage of the mitochondrial respiratory chain complexes in HUVECs. The results of the current study may enhance understanding of the effects of Ang II/atorvastatin on the proliferation of HUVECs and further provide potential therapeutic or preventive targets for hypertension and atherosclerosis.

\section{Acknowledgements}

This study was supported by National Natural Science Foundation of China (grant no. 81470417) and Natural Science Foundation of Liaoning Province (grant no. 2013021090).

\section{References}

1. Whaley-Connell A, Johnson MS and Sowers JR: Aldosterone: role in the cardiometabolic syndrome and resistant hypertension. Prog Cardiovasc Dis 52: 401-409, 2010.

2. Aroor AR, Demarco VG, Jia G, Sun Z, Nistala R, Meininger GA and Sowers JR: The role of tissue renin-angiotensin-aldosterone system in the development of endothelial dysfunction and arterial stiffness. Front Endocrinol (Lausanne) 4: 161, 2013.

3. Schiffrin EL and Touyz RM: From bedside to bench to bedside: role of renin-angiotensin-aldosterone system in remodeling of resistance arteries in hypertension. Am J Physiol Heart Circ Physiol 287: H435-H446, 2004.

4. Touyz RM: The role of angiotensin II in regulating vascular structural and functional changes in hypertension. Curr Hypertens Rep 5: 155-164, 2003.

5. Stoll M, Steckelings UM, Paul M, Bottari SP, Metzger R and Unger T: The angiotensin AT2-receptor mediates inhibition of cell proliferation in coronary endothelial cells. J Clin Invest 95: 651-657, 1995.

6. Sata M and Fukuda D: Crucial role of renin-angiotensin system in the pathogenesis of atherosclerosis. J Med Invest 57: 12-25, 2010.

7. Hill BG, Dranka BP, Zou L, Chatham JC and Darley-Usmar VM: Importance of the bioenergetic reserve capacity in response to cardiomyocyte stress induced by 4-hydroxynonenal. Biochem J 424: 99-107, 2009.

8. Ballinger SW: Mitochondrial dysfunction in cardiovascular disease. Free Radic Biol Med 38: 1278-1295, 2005.

9. Madamanchi NR and Runge MS: Mitochondrial dysfunction in atherosclerosis. Circ Res 100: 460-473, 2007.

10. Ryan MT and Hoogenraad NJ: Mitochondrial-nuclear communications. Annu Rev Biochem 76: 701-722, 2007.

11. Picard M, Taivassalo T, Gouspillou G and Hepple RT: Mitochondria: isolation, structure and function. J Physiol 589: 4413-4421, 2011.

12. Dranka BP, Hill BG and Darley-Usmar VM: Mitochondrial reserve capacity in endothelial cells: the impact of nitric oxide and reactive oxygen species. Free Radic Biol Med 48: 905-914, 2010.

13. Davignon J: Beneficial cardiovascular pleiotropic effects of statins. Circulation 109 (Suppl 1): III39-III43, 2004.

14. Guimarães DA, Rizzi E, Ceron CS, Pinheiro LC, Gerlach RF and Tanus-Santos JE: Atorvastatin and sildenafil lower blood pressure and improve endothelial dysfunction, but only atorvastatin increases vascular stores of nitric oxide in hypertension. Redox Biol 1: 578-585, 2013.

15. Zhou MS, Tian R, Jaimes EA and Raij L: Combination therapy of amlodipine and atorvastatin has more beneficial vascular effects than monotherapy in salt-sensitive hypertension. Am J Hypertens 27: 873-880, 2014

16. Ma Y, Chen Z, Zou Y and Ge J: Atorvastatin represses the angiotensin 2-induced oxidative stress and inflammatory response in dendritic cells via the PI3K/Akt/Nrf2 pathway. Oxid Med Cell Longev 2014: 148798, 2014

17. Chen S, Liu B, Kong D, Li S, Li C, Wang H and Sun Y: Atorvastatin calcium inhibits phenotypic modulation of PDGF-BB-induced VSMCs via down-regulation the Akt signaling pathway. PLoS One 10: e0122577, 2015

18. Ke N, Wang X, Xu X and Abassi YA: The xCELLigence system for real-time and label-free monitoring of cell viability. Methods Mol Biol 740: 33-43, 2011.

19. Ma M, Guo X, Chang Y, Li C, Meng X, Li S, Du ZX, Wang HQ and Sun Y: Advanced glycation end products promote proliferation and suppress autophagy via reduction of cathepsin D in rat vascular smooth muscle cells. Mol Cell Biochem 403: 73-83, 2015.

20. Li Z, Berk M, McIntyre TM, Gores GJ and Feldstein AE: The lysosomal-mitochondrial axis in free fatty acid-induced hepatic lipotoxicity. Hepatology 47: 1495-1503, 2008.

21. Gottlieb E, Armour SM, Harris MH and Thompson CB: Mitochondrial membrane potential regulates matrix configuration and cytochrome $c$ release during apoptosis. Cell Death Differ 10: 709-717, 2003. 
22. Lei T, Guo N, Tan MH and Li YF: Effect of mouse oocyte vitrification on mitochondrial membrane potential and distribution. J Huazhong Univ Sci Technolog Med Sci 34: 99-102, 2014.

23. Hill BG, Higdon AN, Dranka BP and Darley-Usmar VM: Regulation of vascular smooth muscle cell bioenergetic function by protein glutathiolation. Biochim Biophys Acta 1797: 285-295, 2010.

24. Alvarez E, Rodiño-Janeiro BK, Ucieda-Somoza R and González-Juanatey JR: Pravastatin counteracts angiotensin II-induced upregulation and activation of NADPH oxidase at plasma membrane of human endothelial cells. J Cardiovasc Pharmacol 55: 203-212, 2010.

25. Ferrick DA, Neilson A and Beeson C: Advances in measuring cellular bioenergetics using extracellular flux. Drug Discov Today 13: 268-274, 2008.

26. Sansbury BE, Jones SP, Riggs DW, Darley-Usmar VM and Hill BG: Bioenergetic function in cardiovascular cells: the importance of the reserve capacity and its biological regulation. Chem Biol Interact 191: 288-295, 2011.

27. LiP, GuoX,LeiP,ShiS,Luo S and Cheng X: PI3K/Akt/uncoupling protein 2 signaling pathway may be involved in cell senescence and apoptosis induced by angiotensin II in human vascular endothelial cells. Mol Biol Rep 41: 6931-6937, 2014.

28. Eelen G, de Zeeuw P, Simons M and Carmeliet P: Endothelial cell metabolism in normal and diseased vasculature. Circ Res 116: 1231-1244, 2015.

29. Graham DJ, Staffa JA, Shatin D, Andrade SE, Schech SD, La Grenade L, Gurwitz JH, Chan KA, Goodman MJ and Platt R: Incidence of hospitalized rhabdomyolysis in patients treated with lipid-lowering drugs. JAMA 292: 2585-2590, 2004.
30. Sathasivam S: Statin induced myotoxicity. Eur J Intern Med 23 317-324, 2012.

31. Mabuchi H, Higashikata T, Kawashiri M, Katsuda S, Mizuno M, Nohara A, Inazu A, Koizumi J and Kobayashi J: Reduction of serum ubiquinol-10 and ubiquinone-10 levels by atorvastatin in hypercholesterolemic patients. J Atheroscler Thromb 12: 111-119, 2005.

32. Vaughan RA, Garcia-Smith R, Bisoffi M, Conn CA and Trujillo KA: Ubiquinol rescues simvastatin-suppression of mitochondrial content, function and metabolism: implications for statin-induced rhabdomyolysis. Eur J Pharmacol 711: $1-9,2013$

33. Mullen PJ, Zahno A, Lindinger P, Maseneni S, Felser A, Krähenbühl S and Brecht K: Susceptibility to simvastatin-induced toxicity is partly determined by mitochondrial respiration and phosphorylation state of Akt. Biochim Biophys Acta 1813: 2079-2087, 2011.

34. Carnicka S, Adameova A, Nemcekova M, Matejikova J, Pancza D and Ravingerova T: Distinct effects of acute pretreatment with lipophilic and hydrophilic statins on myocardial stunning, arrhythmias and lethal injury in the rat heart subjected to ischemia/reperfusion. Physiol Res 60: 825-830, 2011.

This work is licensed under a Creative Commons Attribution-NonCommercial-NoDerivatives 4.0 International (CC BY-NC-ND 4.0) License. 